\title{
Unwarranted variation in health care for children and young people
}

\author{
C Ronny L H Cheung, ${ }^{1}$ J A Muir Gray ${ }^{2}$
}

${ }^{1}$ Deptartment of Paediatrics, St Thomas' Hospital, London, UK. ${ }^{2}$ NHS QIPP Right Care, Department of Health, London, UK

\section{Correspondence to} Dr Ronny Cheung, Department of Paediatrics, St Thomas' Hospital, London SE1 7EH, UK; crcheung@gmail.com

Received 14 March 2012 Revised 26 July 2012 Accepted 30 July 2012

Published Online First 18 August 2012
To cite: Cheung CRLH, Gray JAM. Arch Dis Child 2013, 98, 60-65.

\section{ABSTRACT}

The study of geographical variation in healthcare has moved on since J Allison Glover's seminal study in 1938, and its value in highlighting inequity in access, quality and outcomes is well-established. Study of variation in healthcare for children, however, has proven more difficult due to barriers with data and idiosyncrasies in how we measure outcomes for children and families. This paper is a narrative review of unwarranted variations in healthcare for children, and discusses the potential of variation analysis to help researchers and policy makers improve child health services.

\section{INTRODUCTION}

"Variation is a thief. It robs from processes, products and services the qualities that they are intended to have.... Unintended variation is stealing healthcare blind today."

Don Berwick ${ }^{1}$

The analysis of variation in healthcare practice and outcomes has its roots in child health. J Allison Glover's 1938 study of the geographical variation in rates of tonsillectomy in school-aged children in Britain was the first of its kind, and the concepts underlying it nearly half a century ahead of its time. ${ }^{2}$ In it, he demonstrated a tenfold variation in tonsillectomy rates in children by education authority-variation that was not correlated with deprivation or any other likely indices of need. $\mathrm{He}$ concluded that the variation 'defies any explanation, save that of variation in medical opinion on the indications for operation, 2

The landscape of healthcare has changed since Glover's time, but variation in healthcare for children remains. Two recent NHS publications have highlighted that such inequity persists. ${ }^{3} 4$ Seventy years on, a similar study to Glover's demonstrated sevenfold variation in rate of tonsillectomy among Local Authority areas in England from 2000-2005. . For children, from 2007-2010 in England there was a sixfold variation by primary care trust (PCT) in rates of elective tonsillectomy. ${ }^{4}$

One of the key drivers for variation analysis research has been health systems' interest in differential health outcomes and the cost of healthcare. Because of the nature of childhood diseases and the smaller numbers involved, the cost of providing healthcare for an ageing adult population dwarfs any child health budget. It is no surprise then, to find that a Medline search for relevant peerreviewed, health-related articles published in the last 10 years with the term 'area variation' as a keyword or in the title/abstract yielded only nineteen studies which included children in their study population, and only five which focused solely on a population below the age of 18 years. The paucity of systematic evidence in area analysis for child health means that what follows here is a pragmatic, narrative review, and evidence is drawn from studies into both adult and child populations.

The analysis of variation in healthcare remains a controversial topic. Many clinicians and healthcare organisations argue that variation in health services reflects all that is good about patient-centred care. Healthcare that is responsive to differences in patient preference, needs and expectations will necessarily differ in process, and perhaps even outcome, from one service to the next. As a result, when variation is identified, a common response is 'our patients are different'.

\section{VARIATION-WARRANTED OR UNWARRANTED?}

Populations are indeed different, not only in terms of their health needs, but also in terms of cultural and personal values, and attitudes to health and risk. Measuring a population's access and use of services, healthcare processes and health outcomes may therefore demonstrate variation that is justifiable and necessary. It may also reflect the adoption of innovations in practice. For these reasons, uniformity of all services is neither possible nor always desirable.

Such is the magnitude of variation seen in recently published data across a range of indicators of healthcare performance and outcome in England; it becomes apparent that patient choice cannot be the sole explanation. ${ }^{3}{ }^{4}$ This premise is strengthened by the fact that, for the majority of those indicators that measured access and quality of service and health outcomes in those publications, study populations were standardised for age and socioeconomic deprivation for more meaningful comparison. ${ }^{4}$

Wennberg coined the term 'unwarranted variation' to describe variation which 'cannot be explained by variation in patient illness or patient preferences."

The challenge is to separate the desirable, patient-centred aspects from those that have a negative impact on children and families due to deficiencies in the delivery of healthcare. As Al Mulley put it: 'If all variation were bad, solutions would be easy. The difficulty is in reducing the bad variation, which reflects the limits of professional knowledge and failures in its application, while preserving the good variation that makes care patient centred."7

Variation in health outcomes and utilisation of health care can be subdivided into three categories: variation in effective, preference-sensitive and supply-sensitive care. ${ }^{6}$ 


\section{VARIATION IN EFFECTIVE (OR HIGH VALUE) CARE}

Variation in effective care, as described by Wennberg, represents variation that exists despite clinical consensus and evidence unequivocally supporting the care process. However, as almost all care is effective to some degree, for certain sub-groups or under certain conditions, we would advocate a change in terminology to 'variation in high value care'. 'High value' care describes care that is effective for the overwhelming majority under any circumstances, variation from which has a detrimental effect on value to both the population and the health care system. Examples include variation in rates of childhood immunisations (optimal level being 100\%) and in rates of diabetic ketoacidosis in children (optimal level being $0 \%$ ).

Because the evidence is clear, when tackling these variations the emphasis is on improving systems of health care delivery, and less so on the need to change perceptions and attitudes among those responsible for health care provision. And simply increasing resources may not be the solution: US studies show no correlation between provision of effective care of long-term conditions and levels of per capita spending, hospital capacity or doctors per capita. ${ }^{8}$

Because of the smaller populations involved and the relative complexity of the spectrum of childhood disease, child health in the UK has led the way for providing high quality care based on managed care networks. Regional managed networks, based around specialist services which provides clinical expertise, ongoing support and education for local providers within their area, have been successful in improving outcomes in diabetes, epilepsy and cystic fibrosis, amongst others. ${ }^{9}{ }^{10}$ Neonatal care has been transformed through neonatal networks, and, importantly, the willingness of clinicians to provide data through the comprehensive national neonatal audit programme for benchmarking and service improvement as well as academic and health services research. ${ }^{11}$ Variation analysis allows commissioners to highlight areas of high variation in outcome or expenditure, and allows providers to benchmark both within and among networks to ensure equity of care.

However, startling variations persist in spite of the strength of evidence and consensus that exist over what constitutes high value care. Among PCTs in England in 2010/11, rates of completion of immunisation with the combined 5-in-1 diphtheria, tetanus, pertussis, polio and Haemophilus influenza type b vaccine (DTaP/IPV/ $\mathrm{Hib})$ at the age of 2 ranges from $85.3 \%$ to $99.6 \%$, meaning that there is an eighteenfold variation in the proportion who have failed to be immunised (see figure 1a and 1b). ${ }^{4}$ In 2009/10, the proportion of known diabetic children aged $0-15$ who were admitted to hospital for diabetic ketoacidosis varied sevenfold among PCTs in England (see figure 2a and 2b). ${ }^{4}$ Variation in high value care, where there is clinical and evidential consensus on best practice and outcomes, highlights systems failures, and allows commissioners and providers to leverage system-wide change, whether that is through creating and improving clinical pathways, promoting adherence to best practice guidance or targeted training of healthcare professionals within the pathway.

\section{VARIATION IN PREFERENCE-SENSITIVE CARE}

Preference-sensitive care describes interventions where the optimal course of action is not as clear. This may be because the evidence is equivocal, or because the thresholds for intervention are subjective (or based solely on consensus). In this situation, the balance of risk and benefit for an intervention may be less clear. Examples in children include the diagnosis and treatment of allergies, or elective tonsillectomy. Variation of this type may also account in part for the fivefold variation seen among PCTs in England for rates of diagnostic gastrointestinal endoscopy in 2007-2010 (see figure 3a and 3b). ${ }^{4}$

Because of the ambiguity in the evidence for preferencesensitive care, and historical and cultural models of patientdoctor relationships, children and families will often defer to their clinician's judgment for the final decision on whether to undergo an intervention, or to pursue an alternative (such as watchful waiting, lifestyle modification or other medical or surgical course of action). The decision will depend on the clinician's interpretation of ambiguous evidence, comfort with a particular technical intervention, or personal assumptions about the impact which the condition or intervention will have on the individual child and family. It is also influenced by the individual clinician's prior experience, personal perspectives on risk tolerance, and personal values. ${ }^{2}$ Because of this dependence on local clinical habits, local patterns of variation tend to remain relatively constant over time-what Wennberg describes as the 'medical/surgical signature. ${ }^{13}$

A clinical encounter in which decision-making is shared between the clinician and a well-informed child and/or family may hold the key to reducing unwarranted variation in preference-sensitive care. A systematic review of patient decision aids has shown that they can result in decision-making that is more in tune with patient preference and, interestingly, results in a reduction in demand for more invasive interventions. ${ }^{14}$ Although it is often argued that shared decision making is difficult to achieve for children, for economic and quality of care reasons, shared decision making for children and families is a promising area of research which is already beginning to reward investment. ${ }^{15} \mathrm{~A}$ retrospective study of children with special healthcare needs has shown that an increase in shared decision making processes was significantly correlated to decreasing cost and utilisation of healthcare services. ${ }^{16}$

\section{VARIATION IN SUPPLY-SENSITIVE CARE}

Variation in supply-sensitive care describes how rates of health care utilisation can be influenced by the availability of local health resources. For instance, the decision of whether or not to admit a patient to hospital, or refer them for an investigation or specialist opinion, depends on a complex set of clinical and nonclinical judgments-not least of which, albeit not consciously, may be the availability of hospital beds, the diagnostic test or access to specialist expertise.

Whatever the cause, such variation in hospital admission rates exists for children living in England today. Emergency admission rates to hospital for children with asthma varies twenty-fivefold by PCT; for epilepsy, ninefold. Rates of admission for bronchiolitis in children varies fourteen-fold, and their length of stay by nearly sixfold-rates which bear no correlation to population indices of socioeconomic deprivation. ${ }^{4}$

Low rates of utilisation may, in theory, represent underprovision of necessary services. Evidence that supports this theory is scanty, ${ }^{17}$ but, given that most of the evidence on variation in supply-sensitive care comes from the US healthcare model, this may be a fruitful area for research in state-funded health systems, especially in these austere times. One example of this is how expanding acute care capacity through primary care-led emergency services has failed to curb attendances to the emergency departments. Rather, attendances have risen, perhaps as a result of absorbing a previously unmet need, or by increasing demand through changing the population perception of need. ${ }^{18}$

More often, though, variation can reveal inappropriately high rates of utilisation which add little value for the population. 
(A)
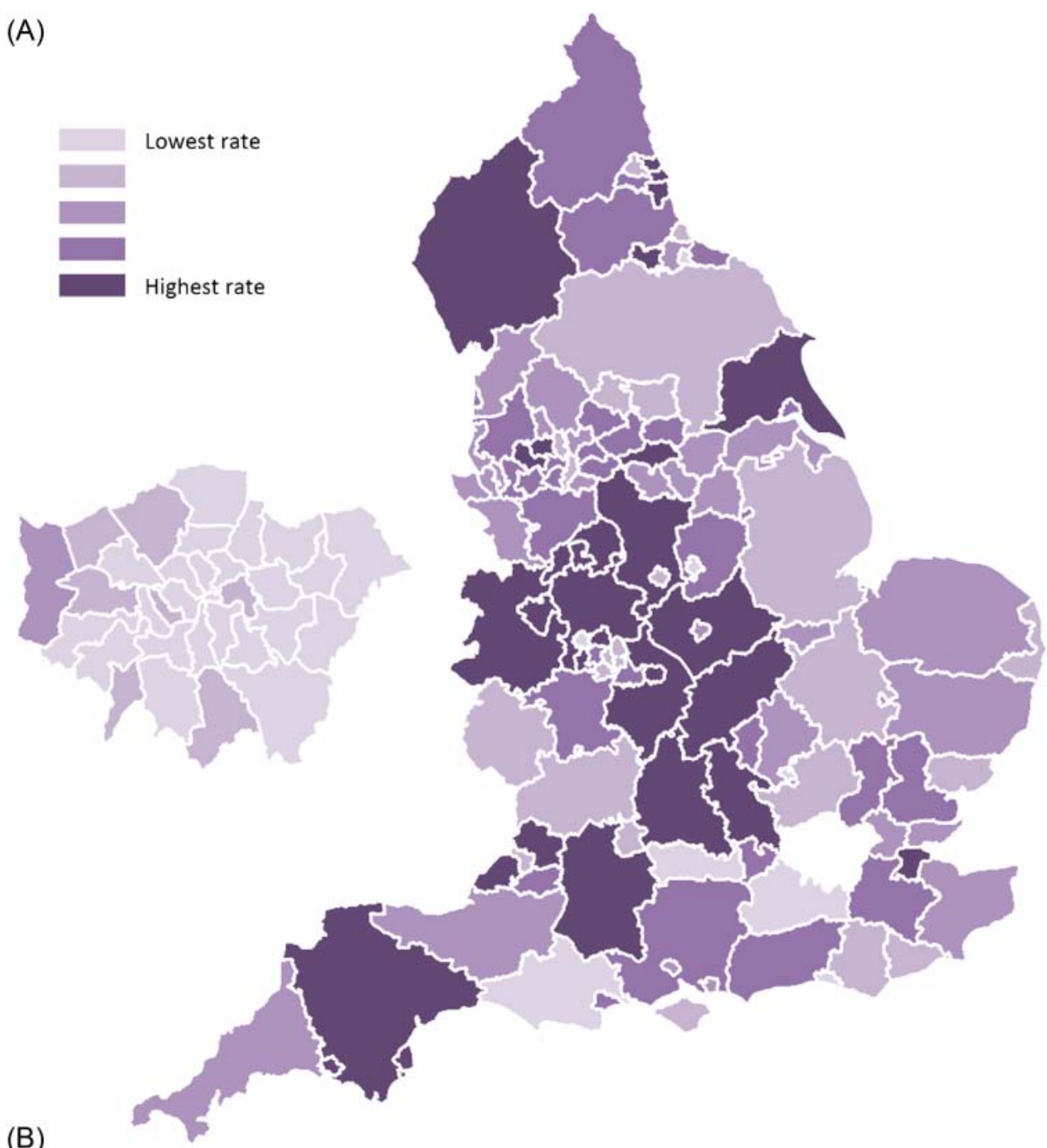

(B)

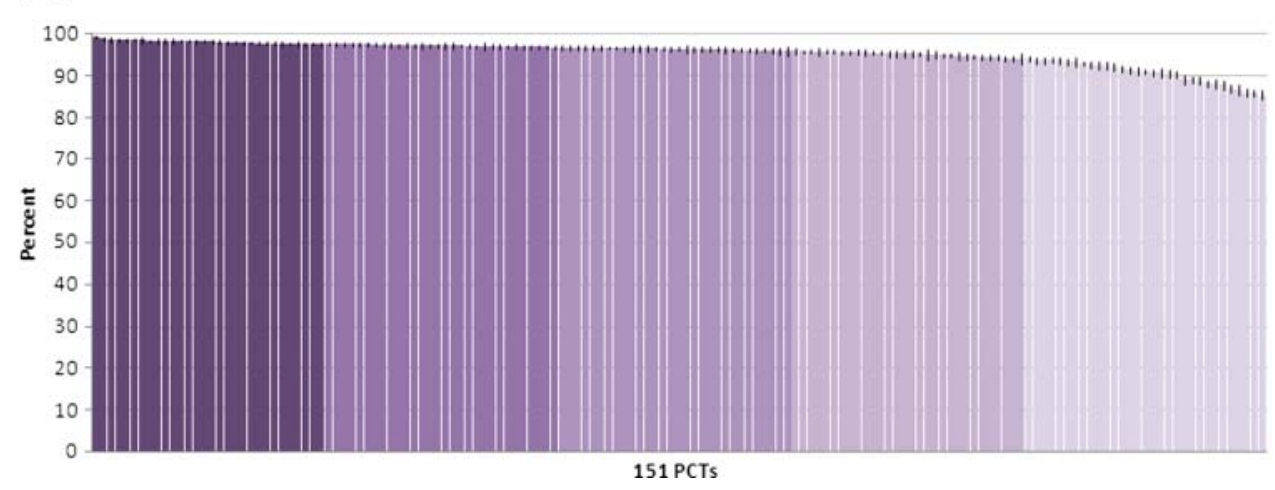

Figure 1 (A) Regional variation in England in the rates of completion for diphtheria, tetanus, pertussis, polio and Haemophilus influenza type b vaccine (DTaP/IPV/Hib) vaccination at the age of 2 years, 2010/11. (B) Variation in England in the percentage completion rate for diphtheria, tetanus, pertussis, polio and Haemophilus influenza type b vaccine (DTaP/IPV/Hib) vaccination at the age of 2 years by primary care trust, 2010/11.

Studies in adult populations show that being treated in a geographical area which has greater resource investment and higher intensity medical input per capita does not always result in appreciable improvements in survival outcomes. ${ }^{19}$ In children, discharge rates from children's wards have shown significant variation which inversely correlates strongly with medical bed capacity, ${ }^{20}$ while variation in the prescribing of stimulant medication for attention deficit hyperactivity disorder has been shown to be positively correlated with supply-side healthcare factors. $^{21}$
Increasing resources will generally provide good value for the population initially. But the increased capacity will begin to drive interventions (such as admissions, or treatments) in those who are less in need. Thus, while the care remains effective, value (to the population as well as to the individual) diminishes. Scarce resource may then be better utilised for another intervention, or a different population altogether.

For the individual too, variation in rates of intervention matter. When healthcare capacity increases, those who are less in need are offered the intervention. For them, the 
(A)

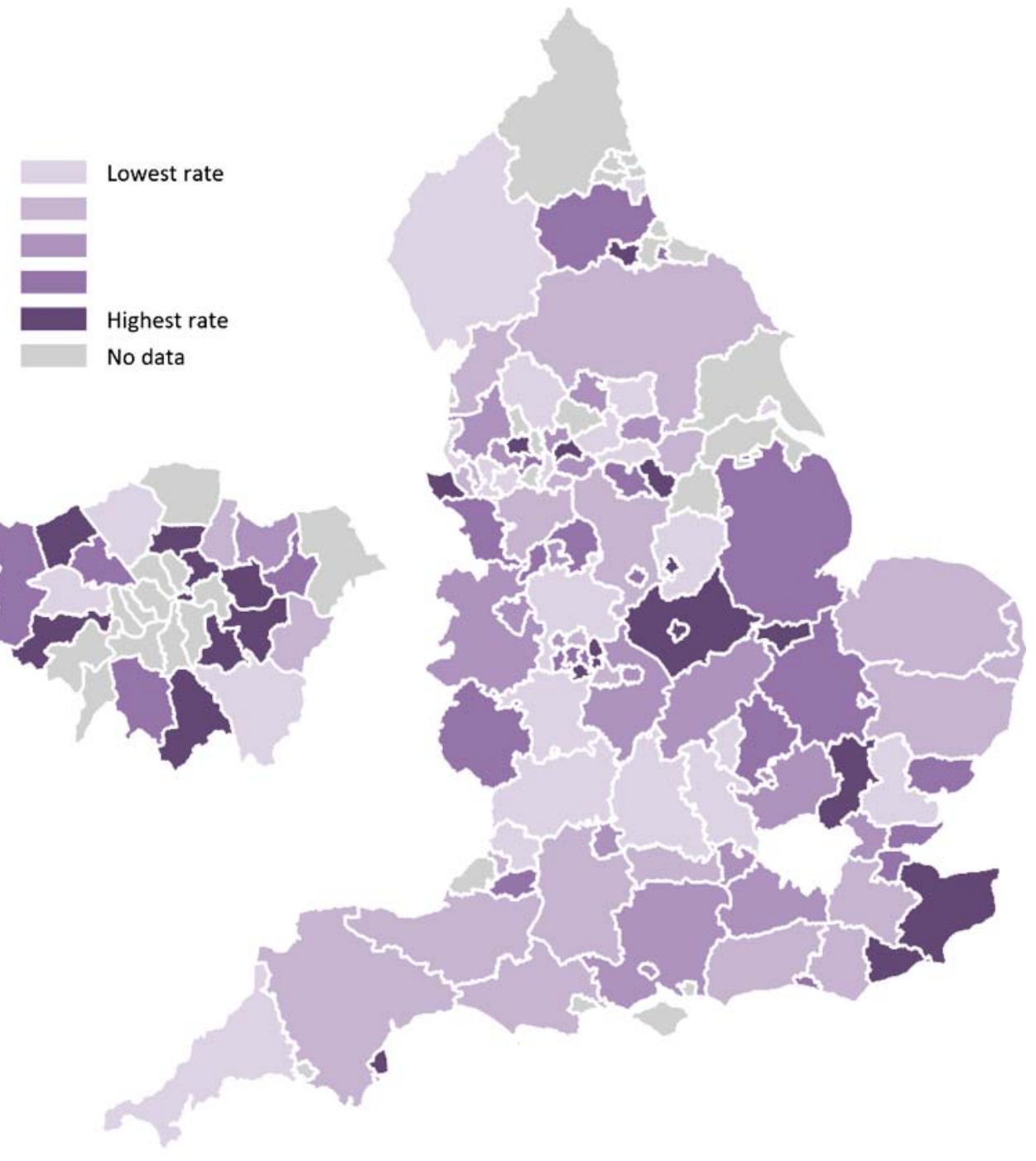

(B)

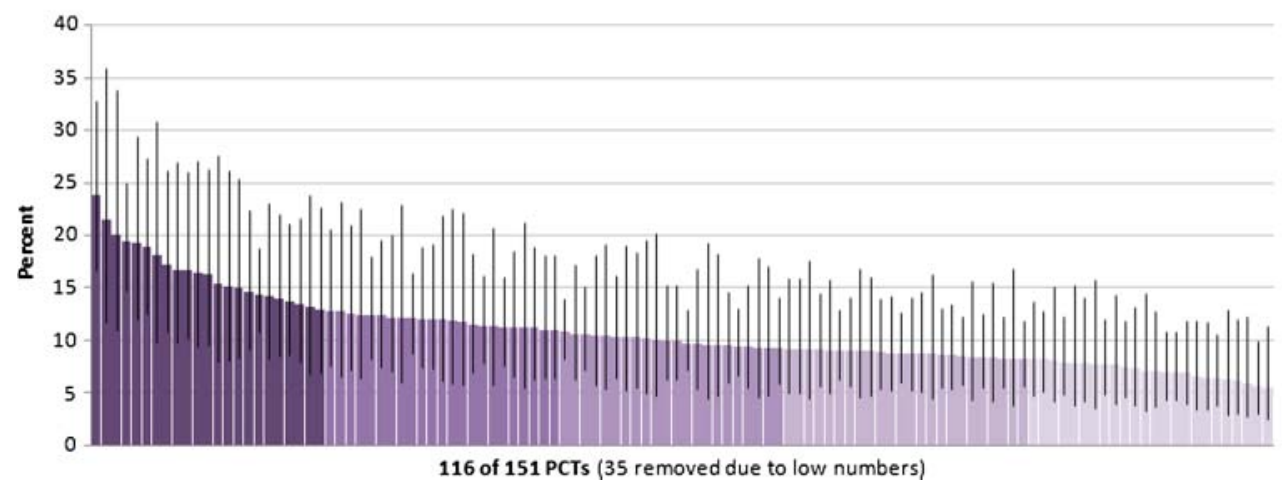

Figure 2 (A) Regional variation in England in the proportion of known diabetic children (aged 0-15 years) who were admitted to hospital for diabetic ketoacidosis during 2009/10. (B) Variation in England in the percentage of known diabetic children (aged 0-15 years) who were admitted to hospital for diabetic ketoacidosis during 2009/10.

magnitude of possible benefit is smaller, but the probability and magnitude of harm from the intervention remains the same. The key for each intervention is to determine the rate at which the ratio of value (for the population as well as for individuals) and harm is greatest-what Donabedian calls the point of optimality $^{\text {,22 }}$

Identifying the point of optimality may be relatively easy to achieve for some conditions. Where disease prevalence is known, and where there are clear criteria for intervention, it may be possible for commissioners to predict, within a margin of error, an expected intervention rate for the local population. This calculated rate could then be compared with actual rates of intervention to identify unmet need or over-utilisation. Where prevalence is unclear, a promising method has been to map a care pathway to identify stages in the patient journey at which variation is most likely to occur, whether that is due to diagnostic uncertainty, variation in thresholds for intervention, or issues of healthcare capacity. ${ }^{23}$ 
(A)

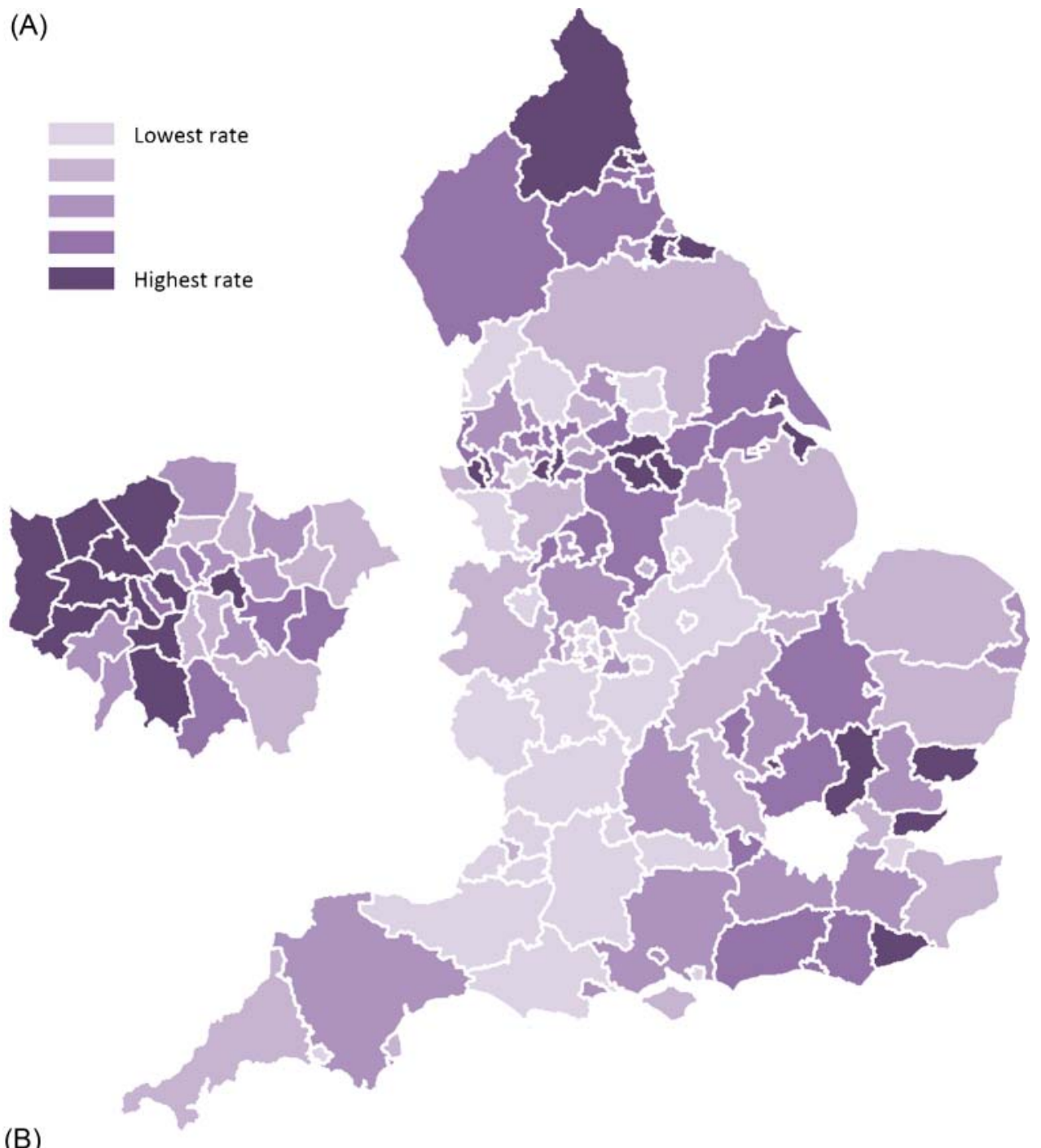

(B)

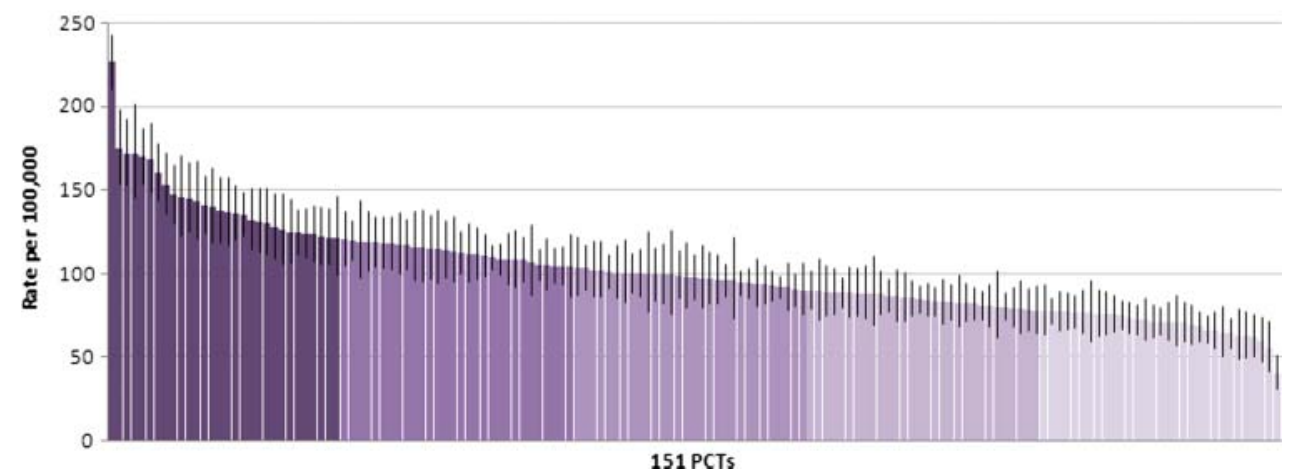

Figure 3 (A) Regional variation in England in the rates of diagnostic upper and/or lower gastrointestinal endoscopy for children aged 0-17 years, 2007-2010. (B) Variation in England in the rate (per 100 000) of diagnostic upper and/or lower gastrointestinal endoscopy for children aged $0-17$ years, 2007-2010.

\section{VALUE AND EQUITY IN CHILD HEALTH AND THE ROLE OF VARIATION ANALYSIS}

Systems variation is a well-established source of waste and inefficiency. Efforts at minimising variation have transformed industrial practices in the past 30 years, ${ }^{24} 25$ and are increasingly embraced by health care organisations and policymakers. ${ }^{26}{ }^{27}$ The current fiscal constraints, from which no country in the world is immune, mean that reducing inefficiencies in health care is a priority for clinicians and commissioners alike. Identifying and reducing unwarranted variation will be key to maximising value-that is, improving productivity and quality to achieve better outcomes with fewer resources. ${ }^{28}$

Value in healthcare can be an unpalatable concept for many clinicians, who pride themselves on advocacy for their own patients. For them, 'value' is seen as a smokescreen for costcutting or rationing, and anathema to clinicians for whom 
clinical effectiveness has traditionally been the only yardstick to measure treatments. But value defined as outcomes relative to $\operatorname{cost}^{28}$ means that cost-cutting which adversely affects quality is counterproductive unless health outcomes improve too. Reducing variation provides value to the NHS through the twin functions of maximising quality and minimising inefficiency.

The role of variation analysis in highlighting equity is a much more familiar concept to child health professionals. Health inequalities in early years have attracted greater prominence in recent years, with increasing appreciation of the disproportionately greater impact which early years interventions may have on later life. ${ }^{29}$ As a result, variation which highlights inequity, both in the state of children's health and in the provision of services, is a cause around which clinicians, politicians and the public can rally.

The solutions though, are not always so easy to implement. There are significant limitations in quality and coverage in existing datasets that are so vital for accurate identification and measurement of variation. ${ }^{4}{ }^{30}$ Rectifying this requires significant central investment or, as exemplified by the national neonatal audit database, outstanding clinical leadership and engagement. ${ }^{11}$ There is also a growing consensus that child health services in this country are fragmented, and that the current balance between accessibility and the quality and safety of child health services is less than optimal. Reconfiguration of services-to concentrate expertise in fewer specialist providers, and for child health services to be provided on a managed clinical network basis which is accountable to larger populationsmakes clinical sense for children, but is a politically sensitive issue, and challenging to implement. The advent of clinical commissioning groups in the NHS in England, which will serve smaller populations than PCTs, means commissioners may find it increasingly difficult to avoid exacerbating existing variations in child health. Analysing variation using different geographical boundaries, as well as mapping how variation changes over time, can be a powerful means by which professionals and commissioners can be held to account for the quality of health care we provide during this period of transition.

Highlighting unwarranted variation can be a lever to change the clinical practices of child health professionals. For policymakers, highlighting existing variation can aid the public to gain a greater understanding of the risks inherent in the existing system, and challenge commissioners to maximise the value, quality and equity of child health services today.

Acknowledgements The authors would like to thank Helen Duncan, Helen Smith and Stephen Chaplin at ChiMat, and Erica Ison at NHS QIPP Right Care, and Andrew Hughes, Caroline Ridler and Kevin Watson at Solutions for Public Health for their help in analysing and presenting data for the graphics shown here. Images are reproduced by kind permission of NHS QIPP Right Care (Crown copyright 2012).

Contributors RC had the idea for the paper and performed the literature search. $\mathrm{RC}$ and JAMG both contributed equally to the development of the paper from first draft to completion. RC is the guarantor.

Funding This work received no specific funding.

Competing interests RC is the editor of the NHS Atlas of Variation in Health Care for Children and Young People (March 2012). JAMG is joint lead for the NHS QIPP Right Care Programme, and co-editor of the NHS Atlas of Variation in Health Care 2010 and 2011.

Provenance and peer review Not commissioned; externally peer reviewed.

\section{REFERENCES}

1 Berwick DM. Controlling variation in health care: a consultation from Walter Shewhart. Med Care 1991;29:1212-25.
2 Glover JA. The incidence of tonsillectomy in school children. Proc $R$ Soc Med 1938;31:95-112.

3 QIPP Right Care. NHS atlas of variation in healthcare: reducing unwarranted variation to increase value and improve quality. London: QIPP Right Care, 2011. www.rightcare.nhs.uk/atlas (accessed 10 July 2012).

4 Cheung R (Ed.) NHS Atlas of Variation in Healthcare for Children and Young People. London: QIPP Right Care, March 2012. www.rightcare.nhs.uk/index.php/ atlas/children-and-young-adults/ (accessed 10 July 2012).

5 Suleman M, Clark MPA, Goldacre M, Burton M. Exploring the variation in paediatric tonsillectomy rates between English regions: a 5-year NHS and independent sector data analysis. Clin Otolaryngol 2010;35:111-17.

6 http://www.dartmouthatlas.org/ (accessed 10 July 2012).

7 Mulley AJ. Improving productivity in the NHS. BMJ 2010;341:c3965. doi:10.1136/ bmj.c3965

8 Wennberg JE, Fisher ES, Goodman DC, et al. Tracking the care of patients with severe chronic illness: the Dartmouth Atlas of Health Care, 2008. www. dartmouthatlas.org/downloads/atlases/2008_chronic_care_atlas.pdf (last accessed 10 July 2012).

9 Greene A, Pagliari C, Cunningham S, et al. Do managed clinical networks improve quality of diabetes care? Evidence from a retrospective mixed methods evaluation. Qual Saf Health Care 2009;18:456-61.

10 NHS Scotland National Services Division. Review of nine national managed clinical networks. NHS Scotland, 2010. http://www.nsd.scot.nhs.uk/publications/ servicereviews/nmen_review.pdf (last accessed 10 July 2012).

11 Spencer A, Modi N. National neonatal data to support specialist care and improve infant outcomes. Arch Dis Child 2012. doi:10.1136/archdischild-2011-300872

12 Eisenberg JM. Doctors' decisions and the cost of medical care: the reasons for doctors' practice patterns and ways to change them. Chicago: Health Administration Press, 1986.

13 Wennberg JE. Tracking medicine. A researcher's quest to understand health care. Oxford: University Press, 2010.

14 O'Connor AM, Bennett CL, Stacey D, et al. Decision aids for people facing health treatment or screening decisions. Cochrane Database Syst Rev 2009;3:CD001431.

15 Butz AM, Walker JM, Pulsifer M, Winkelstein M. Shared Decision Making in School Age Children with Asthma. Pediatr Nurs 2007;33:111-16.

16 Fiks AG, Mayne S, Localio AR, et al. Shared decision-making and health care expenditures among children with special health care needs. Pediatrics 2012;129:99-107.

17 Goodman DC. Unwarranted variation in pediatric medical care. Pediatr Clin N Am 2009;56:745-55

18 Cooke M, Fisher J, Dale J, et al. Reducing attendances and waits in emergency departments. A systematic review of present innovations. National Coordinating Centre for NHS Service Delivery and Organisation, 2004. http://www.sdo.nihr.ac.uk/ files/project/29-final-report.pdf (last accessed 10 July 2012).

19 Wennberg JE. Time to tackle unwarranted variations in practice. BMJ 2011;342:687-90.

20 Goodman DC, Fisher ES, Gittelsohn A, et al. Why are children hospitalized? The role of nonclinical factors in pediatric hospitalizations. Pediatrics 1994;93:896-902.

21 Bruckner TA, Hodgson A, Mahoney $C B$, et al. Health care supply and county-level variation in attention-deficit hyperactivity disorder prescription medications. Pharmacoepidemiol Drug Saf 2011. doi:10.1002/pds.2264

22 Donabedian A. An introduction to quality assurance in healthcare. Oxford: Oxford University Press, 2002.

23 Bohm N, Swift S, Ceney A, Birchall M. Regional and local variation of ventilation tube insertion in England: a quantitative analysis. Oral presentation at 11 th International congress of European Society of Paediatric Otolaryngology 2012, 22nd May 2012.

24 Deming WE. Out of the Crisis. MIT Center for Advanced Engineering Study, 1983

25 Womack JP, Jones DT, Roos D. The machine that changed the world: the story of lean production. New York, USA: Harper Perennial, 1991.

26 NHS Confederation. Variation in health care: does it matter and can anything be done? London: NHS Confederation, 2004.

27 Appleby J, Ham C, Imison C, Jennings M. Improving NHS productivity: more with the same not more of the same. London: King's Fund, 2010.

28 Porter M. What is value in health care? NEJM 2010;363:2477-81.

29 Marmot M. Fair society, healthy lives. Strategic review of health inequalities in England post 2010. London: Marmot Review, 2010.

30 Kennedy I. Getting it right for children and young people: Overcoming cultural barriers in the NHS so as to meet their needs. London: Stationery Office, 2010.

31 RCPCH. Facing the future: a review of paediatric services. London: Royal College of Paediatrics and Child Health, 2010. www.rcpch.ac.uk/facingthefuture (accessed 10 July 2012).

32 Wolfe I, Cass $\mathrm{H}$, Thompson $\mathrm{M}$, et al. Improving child health services in the UK: insights from Europe and their implications for the NHS reforms. BMJ 2011;342: d1277. 